\title{
Environmental Adaptation of calcification from coccolith associated polysaccharides
}

ROSALIND E. M. RICKABY ${ }^{1}$, LOUIS M. CLAXTON ${ }^{1}$ AND RENEE B. Y. LEE ${ }^{2}$

${ }^{1}$ University of Oxford

${ }^{2}$ University of Reading

Presenting Author: rosr@earth.ox.ac.uk

During their lith formation, coccolithophores mainly employ coccolith-associated polysaccharides (CAPs) for the regulation of crystal nucleation and growth. Different species of coccolithophore dedicate varying proportions of their total photosynthetic budget, ranging from $0.2 \%$ to $7 \%$, towards the control of calcification [1]. Within species, it is totally unknown how that energetic investment in calcification regulation varies with carbonate chemistry and whether such different cellular resource allocation strategies may affect susceptibility to malformation and acidification.

The CAP macromolecules interact with the intracellular calcifying compartment (coccolith vesicle) through the charged carboxyl groups of their uronic acid residues. In previous work, we have hypothesised that an inverse correlation between the uronic acid contents of CAPs and the internal saturation state of the coccolith vesicle may exist [2].

Here we will use culture experiments of Coccolithus pelagicus, and Gephyrocapsa oceanica to investigate the different strategies for control of calcification when confronted by changing carbonate chemistry. We aim to probe the different species strategies in response to varying substrate availability for photosynthesis $(\mathrm{CO} 2)$, versus cost of calcification (saturation state). For each of these species, we will explore the interplay between the proportion of energy from photosynthesis that is invested in the control of calcification versus the chemical control exerted by the uronic acid content of the CAPs to understand their adaptation to the modern and past environments.

[1] Monteiro, F. M., et al., Why marine phytoplankton Calcify? Science Advances, 2 (7) e1501822, 2016

[2] Lee, R. B. Y., et al.,, The Uronic acid content of coccolith associated polysaccharide provides insights into evolving coccolithogenesis and climate, Nat. Comms, doi:10.1038/ncomms13144, 2016 